\title{
Paper
}

\section{Emission Characteristics of High Color Rendering Index Low- Pressure Xe and CO ICP's Light Source}

\author{
Ahmad NAZRI, Akira KONDO, Hideki MOTOMURA and Masafumi JINNO \\ Department of Electrical and Electronic Engineering, Ehime University
}

Received September 28, 2007, Accepted December 16, 2008

\begin{abstract}
High color rendering index (CRI) has been obtained from mercury-free ICP's lamp. Due to the strong demand on the development of mercury-free light sources, the authors investigated xenon and $\mathrm{CO}$ as an alternative element. These rare (Xe) and molecule ( $\mathrm{CO}$ ) gases have been used as a substitute for mercury as the environmental problems related necessitate the development of new type of light sources. The target of this study is to develop a new type of mercury-free lamp without using a phosphor where the authors concentrate on how to obtain a strong visible light instead of UV. In this paper, the colors, luminance and emission characteristics of these types of mercury-free ICP's lamp is discussed. Without the use of phosphor, the authors obtained a good color rendering from xenon and CO ICP's lamp where their CRl's above 90 . A strong continuum emission in visible region is obtained where luminance are ranged from 2000 to $25000 \mathrm{~cd} / \mathrm{m}^{2}$ at $100 \mathrm{~W}$ of input power. Spectral distribution shows the continuum emissions are similar to the daylight distribution and chromaticity diagrams shows they are close to white region in color coordinates and color temperatures bringing a high index of the lamp's color rendering.
\end{abstract}

KEYWORDS: CRI, mercury-free, ICP, Xenon, CO

\section{Introduction}

Nowadays a demand of the global mercury consumption is decreasing because of its toxicity. However mercury is still mainly used such as for industrial batteries, manometers, fluorescent lamps, etc. Mercury is an essential element in the lighting industry as most types of lamps such as low-pressure fluorescent lamps, high pressure HID lamps, metal halide lamps are employing it. Mercury based discharge lamps shows high performance at relatively low price. RoHS') and $\mathrm{WEEE}^{2}$ ) directive that adopted by the European Union (EU) are the latest and detail legislation in dealing with hazardous substances in electrical and electronic equipment. However, there is an exemption in the case of mercury for light sources that poses a restriction in the amount to be used. In detailed, for compact fluorescent lamp it may not exceed $5 \mathrm{mg}$ per lamp and for straight fluorescent lamp it has to be less than $10 \mathrm{mg}{ }^{1)}$. Even though the current legislation treats mercury in light sources in special way, it is important to realize the mercury-free light source is needed in the near future. An alternative to mencury lamp that can be considered is rare and molecule gases based discharge lamp. Gases based discharge lamp also has an advantage in temperature-independence, capable of instant starting and restarting compared to mercury lamps as there is no evaporation process required.
In a fluorescent lamp the use of phosphor is essential in order to convert UV (ultra violet) to visible light. Most of the phosphors are developed exclusive to designated UV wavelengths or elements. With currently available phosphor it is difficult to get a good conversion efficiency from UV to visible light when the used UV wavelength is not matched with the original one especially when new element is used. Moreover, the used of phosphor in lamp itself is costly and to develop a new phosphor for a new lamp will be more costly. Due to this point of view, the authors concentrate on developing a new type of mercuryfree lamp without the use of phosphor, which is concentrate on how to obtain a strong visible light instead of UV emission.

In present conventional discharge such as DC and low frequency $\mathrm{AC}$, the electrodes inside the tube are required to ignite and sustain the discharge. Due to this, the lamp lifetime mostly depends on the duration of electrodes. This makes more frequent maintenance, increases the waste and cost a lot for its disposal system which consequently brings the environmental issue. To overcome this global issue, the development of mercury-free electrodeless discharge light sources is an important and urgent task. The authors have been undergoing the development of mercury-free electrodeless lamp using inductively coupled plasma (ICP). ICP is one of the electrodeless discharges 
which widely used in semiconductor production such for etching and surface processing. ICP has a capability to produce intense and high density plasma. This characteristic can lead to obtain a strong continuum visible emission.

The authors have been using xenon (Xe) and carbon monoxide (CO) as an alternative to mercury. Lowpressure xenon is commonly used as fluorescent lamp because its resonance emission in VUV (vacuum ultra violet) is the nearest one to mercury compared to other rare gases. At high-pressure like HID lamp, xenon can emit a strong continuum visible emission that similar to daylight spectrum which gives an ability to render colors in natural way. In this study, the authors used lowpressure xenon to obtain continuum visible emission instead of high-pressure and without the use of phosphor. For CO, as far as the authors concern, there is no report on it for lighting application, except by $\mathrm{Hatta}^{3 / 4)}$ where $\mathrm{CO}$ was used using cold cathode discharge. Hatta reported that both discharge and emission intensity are unstable which is can be considered due to the reaction between electrodes and CO molecules, and the use of ICP is expected can overcome this problem.

Moreover in order to obtain an instant starting and restarting lamp with a constant light quantity, the authors focus on gas-based discharge. As an initial step, xenon (rare) and CO (molecule) gases are used due to their characteristics as discussed above.

\section{Experimental Setup}

\subsection{Discharge tube and ICP circuit}

The schematic diagram of the lamp (cylindrical tube) used in the experiment is shown in Figure 1. The inner diameter of the lamp is $26 \mathrm{~mm}$ and outer diameter is 30 $\mathrm{mm}$. Two types of lamps were made with one is rare gas xenon lamp and the other one is molecule gas CO lamp. The lamps were baked and after filled with gases. In case of xenon lamp, 5 different pressures which ranged from 0.2 Torr (26.6 Pa) to 50 Torr $(6.65 \mathrm{kPa})$ were used. For CO lamp, 3 different pressures at 0.05 Torr $(6.65 \mathrm{~Pa}), 0.1 \mathrm{Torr}$ (13.3 Pa) and 1 Torr $(133 \mathrm{~Pa})$ were tested. The lamp which is made from Pyrex glass was wound with 4-turn of copper wire (1.6 $\mathrm{mm}$ in diameter) with $10 \mathrm{~mm}$ of winding gap. This copper wire is used as an antenna coil for inductive discharge. The high frequency power supply (Cesar 1310) with frequency $13.56 \mathrm{MHz}$ was generated and supplied to the lamp through matching network. Figure 2 shows the equivalent circuit used in the experiment. $R_{\mathrm{T}}$ is resistance of power supply, $R_{\mathrm{p}}$ and $L_{\mathrm{p}}$ represents plasma resistance and inductance respectively. $C_{1}$ and $C_{2}$ are adjustable capacitors with the maximum capacitance of $700 \mathrm{pF}$ and $2000 \mathrm{pF}$ respectively. These capacitors were adjusted to match the impedance and reduce the reflected power. The input power (output

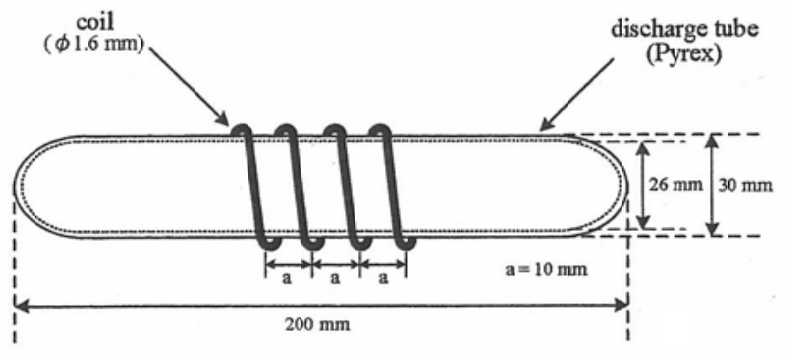

Figure 1 Discharge tube with coil used in the experiment

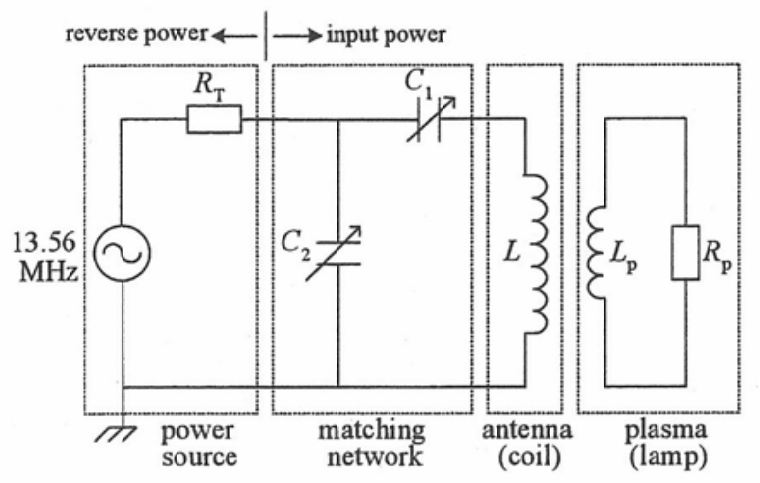

Figure 2 Equivalent circuit of the ICP discharge

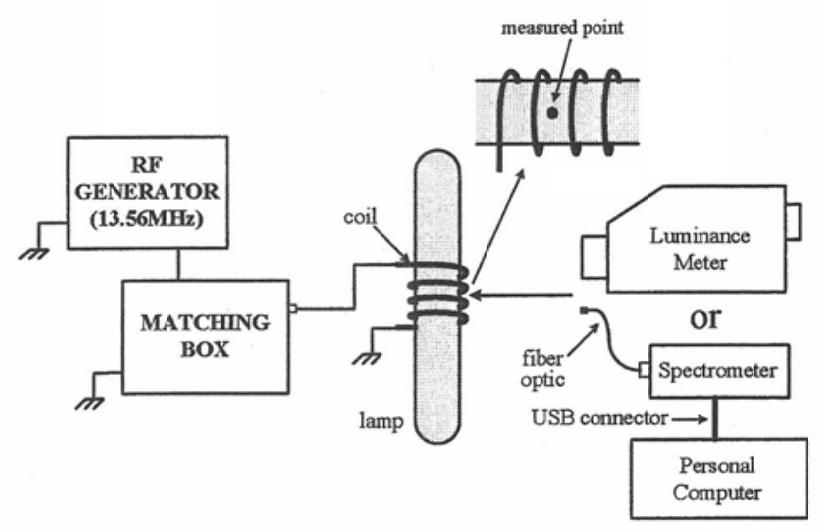

Figure 3 Connection diagram for luminance and spectrum measurements

power from $\mathrm{RF}$ generator when reflected power is $0 \mathrm{~W}$ ) was varied from to 30 to $100 \mathrm{~W}$.

\subsection{Luminance and spectrum measurement}

Diagram of the setup for the luminance and spectrum measurement is given in Figure 3. Measurement point was set at the middle of lamp as shown in the figure. Luminance was measured using Topcon BM-7 luminance meter set at $50 \mathrm{~cm}$ away from measured point with a field angle of $0.2^{\circ}$. The Ocean Optics USB 2000 CCD spectrometer was used to measure the emission spectra. This spectrometer using fiber optic, covers from $200 \mathrm{~nm}$ to $850 \mathrm{~nm}$ with a 600 line/mm grating blazed at $400 \mathrm{~nm}$ and with $10 \mu \mathrm{m}$ of entrance slit. The evaluation was done at 
near UV and visible region. Its spectroscopic sensitivity was calibrated using the Bentham SRS8-Q standard light source. The electron configuration and energy values for lower and upper levels for xenon emission are obtained using Sapphire Professional version 1.0.3.2 electronic spectroscopy software from Cavendish Instruments Ltd.

\subsection{Color Rendering Index (CRI)}

The CRI or $R_{\mathrm{a}}$ is a measure of the degree of change in the apparent colors of standardized pigments perceived when they are illuminated by the sources). Following the CIE (International Commission on Mlumination), color rendering is given as index between 0 and 100, where lower value indicate poor color rendering and higher ones good color rendering. In general, light sources with high CRI (80 to 100) will make people and things look better than those with lower CRIs. In this study, the CRI was derived from emission spectrum and was calculated using BenWint spectro-radiometer version 1.0.1.12, the measurement software from Bentham Instruments Ltd. BenWin + was also used to measure the color coordinate and color temperature.

\section{Results and Discussion}

\subsection{Luminance}

Figure 4 shows luminance result for xenon ICP's lamp when input power is varied. Luminance increases accordingly to the increased of input power. The highest luminance is obtained when gas pressure at 0.2 Torr where the luminance is $11100 \mathrm{~cd} / \mathrm{m}^{2}$. This is due to the one of the advantages of ICP which is can produce high intensity plasma at even a very low pressure. However the luminance is significantly dropped at pressure around 1 Torr and increases when gas pressure is increased. This characteristic which is thought due to the effect of different heating mechanism where was discussed by the authors previouslyo).

Figure 5 shows the luminance results for CO ICP's lamp. At only $\mathrm{CO}$ pressure of 0.05 Torr, the plasma is succeed to produce at $\mathrm{H}$-mode (inductive mode) discharge whereas at 0.1 Torr discharge was unstable and only can be produced at E-mode (capacitive mode) and at 1 Torr the plasma cannot be produced at all. Even at 0.05 Torr also it is hard to sustain the discharge when input power is below $60 \mathrm{~W}$. These are due to high breakdown voltage of $\mathrm{CO}$ molecule which need more input power to ignite and sustain the discharge especially at higher pressure (more than 0.05 Torr). As shown in Figure 5, the highest luminance obtained was approximately $23000 \mathrm{~cd} / \mathrm{m}^{2}$, and can be considered a high luminance value in terms of general domestic purposes.

\subsection{Emission spectra characteristics}

The emission spectra of xenon ICP's lamp in the near

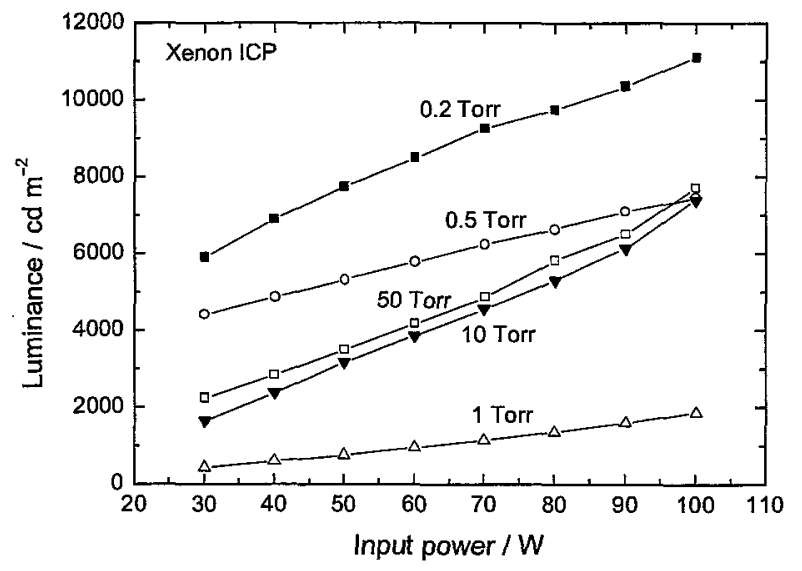

Figure 4 Xenon lamp luminance at different pressure and input power. 0.2 Torr shows the highest luminance.

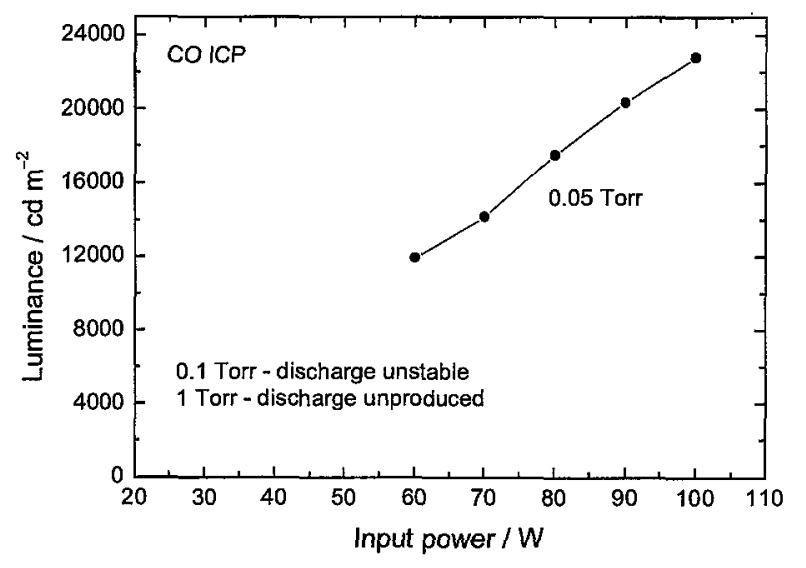

Figure 5 Luminance of 0.05 Torr CO ICP at different input power (for 0.1 and 1 Torr, data cannot be taken due to uncertain plasma condition)

UV and the visible region ( $300 \sim 780 \mathrm{~nm})$ at 0.2 Torr, 1 Torr and 50 Torr are shown in Figures 6, 7 and 8 respectively. Some of the main atomic and ionic lines emitted are indicated. A visible continuum emission can be seen in Figures 6 (0.2 Torr) and 8 (50 Torr) where they are stronger compared to that shown in Figure 7 of low luminance at 1 Torr. This strong visible continuum emission is believed to result mainly from electron-ion collisions that produce recombination radiation and from free-free transitions which produce bremsstrahlung radiation. The spectrum is mainly assigned to xenon atomic lines that belong to transitions originating from $5 p^{5} 6 p\left(2 p_{1}\right.$ to $\left.2 p_{10}\right)$ configuration at energy levels between $9.58 \mathrm{eV}$ and $11.98 \mathrm{eV}$. In Figures 6 and 7, the intensity of some xenon ionic lines are high compared to that in Figure 8, especially for the transitions at $484.56 \mathrm{~nm}$ $\left(6 \mathrm{p}^{4} \mathrm{D}^{0}{ }_{7 / 2} \rightarrow 6 \mathrm{~s}^{4} \mathrm{P}_{5 / 2}, 14.1 \mathrm{eV}\right.$ to $\left.11.54 \mathrm{eV}\right)$, and $529.36 \mathrm{~nm}$ $\left(6 \mathrm{p}^{4} \mathrm{P}_{5 / 2} \rightarrow 6 \mathrm{~s}^{4} \mathrm{P}_{5 / 2}, 13.88 \mathrm{eV}\right.$ to $\left.11.54 \mathrm{eV}\right)$. This result shows that at lower pressures ( 0.2 Torr and 1 Torr) the ionic lines are stronger compared to those at a higher pressure (50 


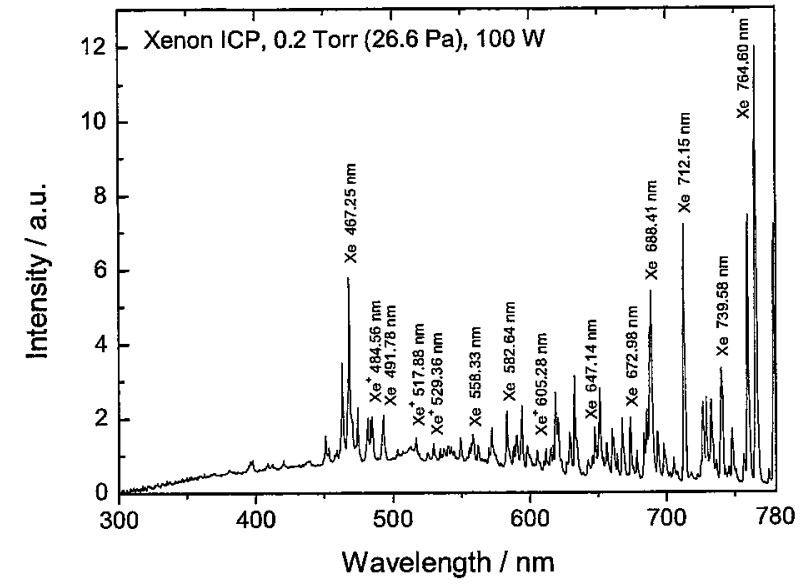

Figure 6 Spectral distribution of xenon ICP at 0.2 Torr, $100 \mathrm{~W}$. Strong continuum visible emission is observed.

Torr). This can be a consequence of the longer mean free path of the electrons at lower pressures which would allow them to accelerate to higher speeds and therefore more energetic collisions with the atoms and ions.

Emission spectrum for CO 0.05 Torr $(300 \sim 780 \mathrm{~nm})$ is shown in Figure 9. As like xenon emission, there is strong continuum visible emission obtained which can be considered mainly from electron-ion recombination process. An intense line spectra at $777.41 \mathrm{~nm}$ which is assigned to atomic emission of $\mathrm{O}$ from $3 p$ orbit $\left(3 p^{5} \mathrm{P}_{3} \rightarrow\right.$ $3 \mathrm{~s}^{5} \mathrm{~S}_{2}, 10.74 \mathrm{eV}$ to $9.15 \mathrm{eV}$ ) is observed. Many $\mathrm{CO}$ molecules are dissociated and can be considered not only from CO molecule itself, but also from fragmented species. Together with continuum emission, the jagged lines $(300 \sim 600 \mathrm{~nm})$ of spectrum also can be observed. At this stage it is difficult to identify every single emission for these jagged lines. However, it is thought to be molecular radiations (from $\mathrm{C}_{2}, \mathrm{O}_{2}$ or any impurities) due to its long wavelength bandwidth.

\subsection{Color rendering}

Figure 10 shows the relation between the CRI and gas pressure for xenon and $\mathrm{CO}$ at input power of $100 \mathrm{~W}$. In case of xenon, the CRI value increases when gas pressure is increased from 1 Torr to 50 Torr. The CRI is getting lower at pressures around 1 Torr and rises again when the gas pressure is decreased from 1 Torr to 0.2 Torr. This is thought also due to different heating mechanisms similar with the luminance characteristic as previously discussed. This heating mechanism differs corresponding to the Ramsauer effect where at a certain electron energy the collision cross-section area becomes lower. When the collision cross-section area decreases, total collision also decreases and less recombination radiation is produced. Consequently continuum emissions in a visible region become lower. The CRI for xenon is at its highest value of

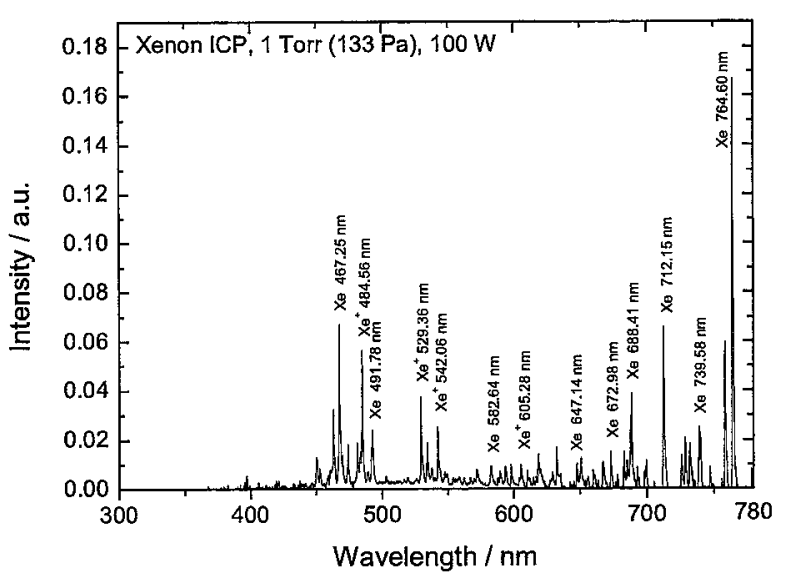

Figure 7 Spectral distribution of xenon ICP at 1 Torr, $100 \mathrm{~W}$. Only atomic and ionic emissions are observed.

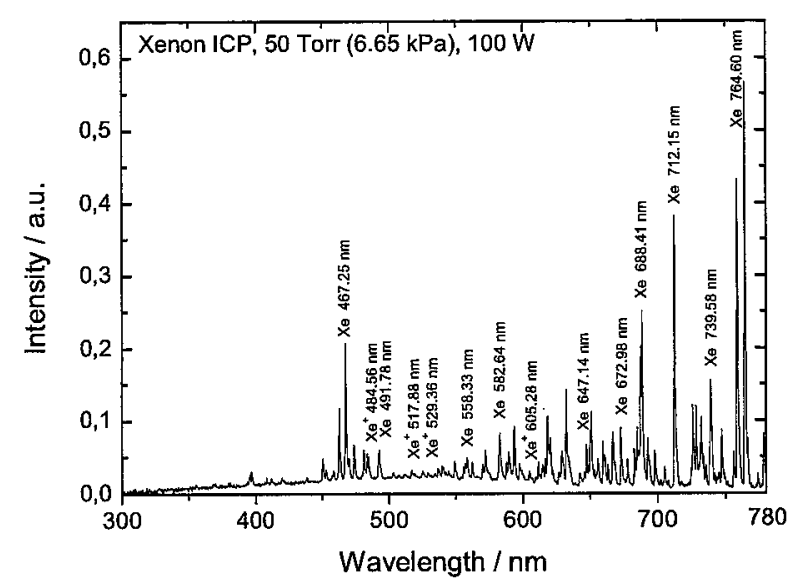

Figure 8 Spectral distribution of xenon ICP at 50 Tor, $100 \mathrm{~W}$. Relatively strong continuum visible emission is observed.

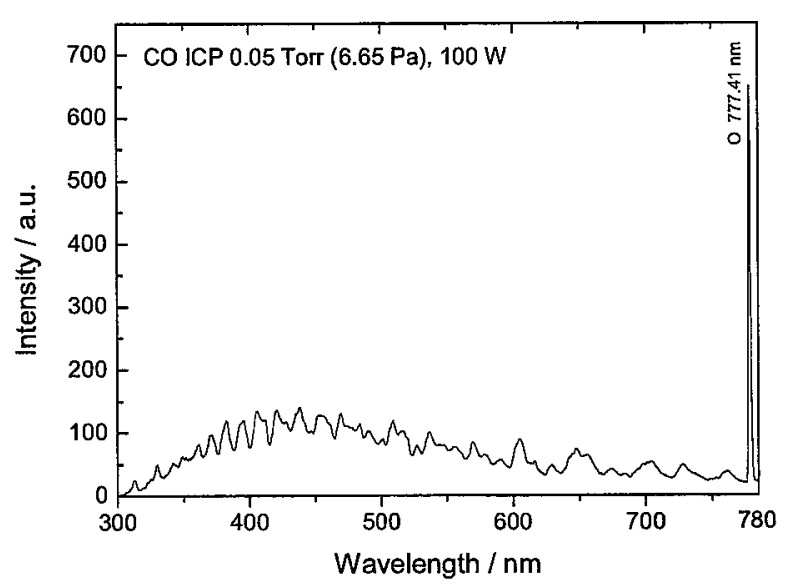

Figure 9 Spectral distribution of CO ICP at 0.05 Tor, $100 \mathrm{~W}$. Strong continuum visible emission and $O$ atomic line is observed. 
95 when the gas pressure is 0.2 Torr. CO ICP lamp also produced a high CRI value at 91 . These values are higher than those characterized as the general fluorescent lamps which around 80. A high CRI shows that the emissions in the red, green and blue regions of the spectrum obtained from xenon and CO ICP's lamp at the above conditions are in a good balance.

As shown in Figures 6, 8 and 9, continuum visible emission is strong and covers the entire visible area. On the other hand, the spectrum in Figure 7 shows a weak continuum emission where it is dominant by line emissions. The luminance and the CRI value of the ICP's lamp are strongly related to this continuum visible emission. Good color rendering means that the lamp is close to the natural daylight. Figure 11 shows the spectrum of the daylight. In the case of xenon 0.2 Torr and CO 0.05 Torr, the continuum emission is similar to the daylight continuum spectrum up to around $650 \mathrm{~nm}$.

Good color rendering also illustrates how 'white' the lamp itself. The more 'white' emission is obtained, the more natural the light is (compared to the daylight). In this context, 'white' is defined by the color coordinates and color temperature. These color coordinate, color temperature and spectral distribution are the main three elements that describe the visual characteristics of the lamp. Color coordinates and color temperature can be expressed using CIE chromaticity diagram where the white region has coordinates of $x$ and $y$ at 0.35 for both axes and with $5000 \mathrm{~K}$ of color temperature. According to CIE chromaticity diagram, as the temperature increases the lamp is generally contain more blue. For example, a black body at normal room temperature will appear black, at $800 \mathrm{~K}$ will be dull red, at $3000 \mathrm{~K}$ will be yellow, at 5000 $\mathrm{K}$ it will be white, pale blue at $8000 \mathrm{~K}$ and a bright blue at $50000 \mathrm{~K} 7$. The color coordinates and color temperature of xenon 0.2 Torr, 1 Torr, 50 Torr and CO 0.05 Torr are shown in Figure 12. It shows that at the highest CRI at xenon 0.2 Torr, it is at its closest to the white region in where the color coordinates are $x=0.31, y=0.33$ and color temperature at $6515 \mathrm{~K}$. The lowest CRI at 63 of xenon 1 Torr shows color coordinates with $x$ and $y$ at 0.29 and 0.34 respectively, and color temperature at $7589 \mathrm{~K}$. In case of $\mathrm{CO}$ even the color rendering is high at 91 , the color coordinate is slightly shifted with $x$ and $y$ coordinates at 0.27 and 0.28 respectively and its color temperature is not in white region at $10647 \mathrm{~K}$ In terms of 'white' color lamp according to CIE diagram, xenon 0.2 Torr can be considered nearly fulfilled the 'white' condition whereas the other two conditions are slightly shifted.

\section{Conclusion}

Luminance and emission characteristics of the xenon and CO ICP's lamp were investigated and their color properties are evaluated. Without the phosphor coating,

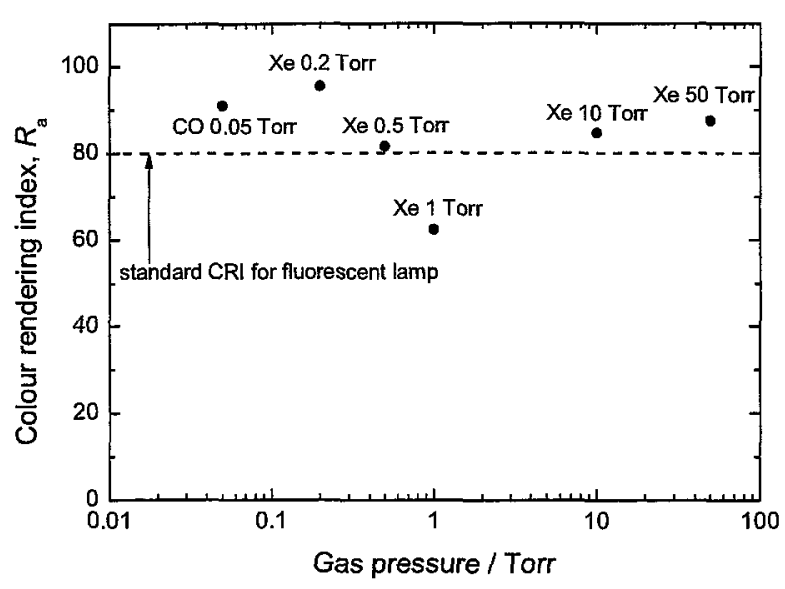

Figure 10 Color rendering index for xenon and $\mathrm{CO}$. Except at xenon 1 Torr, all the value are above the CRI standard for fluorescent lamp.

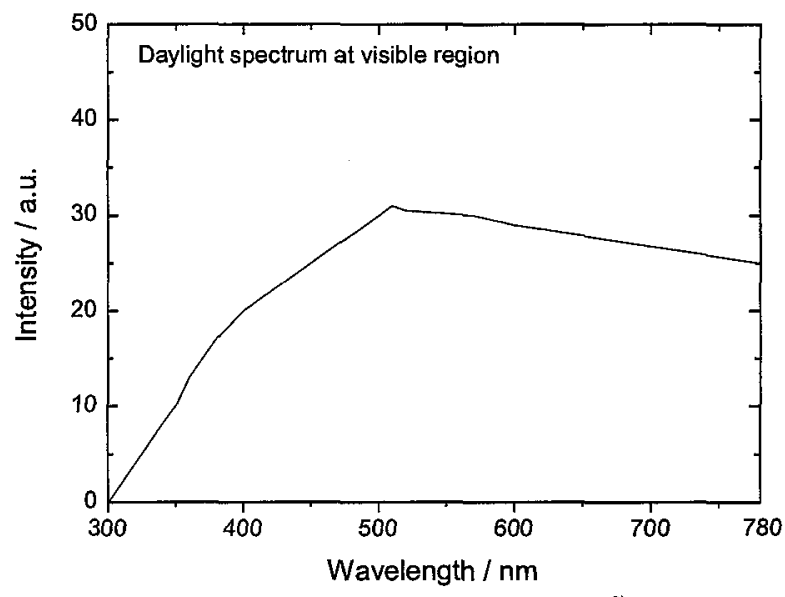

Figure 11 Daylight spectrum at visible region ${ }^{8}$. A continuum visible emission is observed.

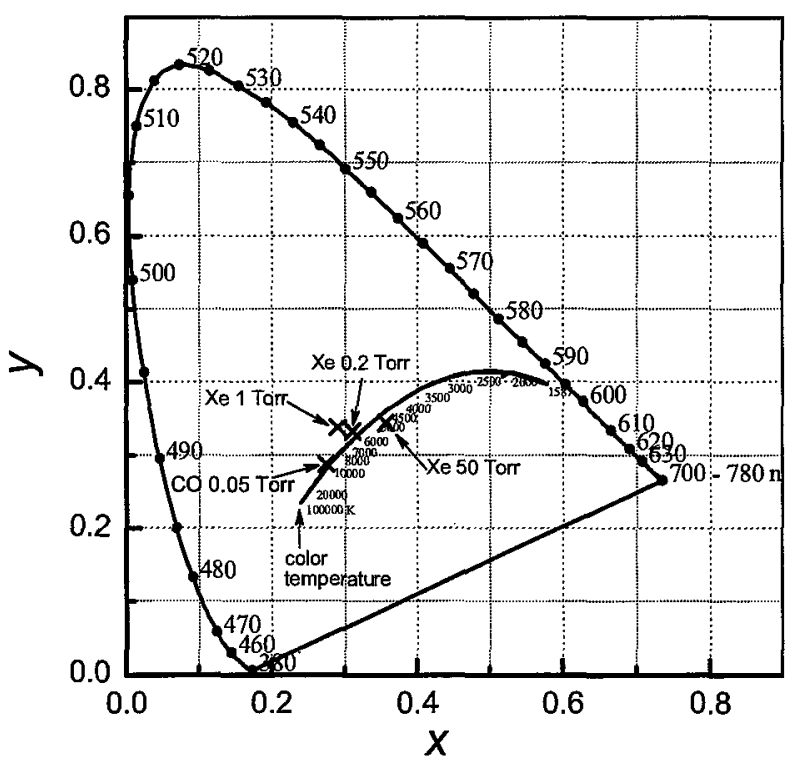

Figure $12 \mathrm{ClE}$ chromaticity diagram for different xenon pressures and $\mathrm{CO}$ at 0.05 Torr 
these mercury-free electrodeless lamps give high luminance and good color rendering especially at xenon 0.2 Torr $\left(12000 \mathrm{~cd} / \mathrm{m}^{2}, \mathrm{CRI}=95\right)$ and CO 0.05 Torr (23000 $\mathrm{cd} / \mathrm{m}^{2}, \mathrm{CRI}=91$ ), which provide a comparative results compared to mercury fluorescent electroded lamp $\left(10000 \sim 20000 \mathrm{~cd} / \mathrm{m}^{2}, \mathrm{CRI}=80 \sim 90\right)$. In addition at xenon 02 Torr, 'white' color lamp has been obtained through evaluation using CIE chromaticity diagram where its color coordinate and color temperature are in white region. These gas-based electrodeless lamps are initial steps towards the goal set which is the development of an efficient mercury-free electrodeless light source and the works in ongoing.

\section{Acknowledgements}

The authors thank technical staff Mr. Hisayoshi Kurokawa for his assistance in lamps making. Part of this work has been supported by Japan Society for Promotion Science (JSPS), Grant-in-Aid for young scientist (A) (14702067).

\section{References}

(1) Directive 2002/95/EC, Restriction of the use of the certain hazardous substances in electrical and electronic equipment, Official Journal of the European Union, Legislation, pp.19-23 (2003).

(2) Directive 2002/96/EC, Waste electrical and electronic equipment, Official Journal of the European Union, Legislation, pp.24-38 (2003).

(3) Hatta, A.: VUV emission from CO gas discharge, Proceedings of the $10^{\text {th }}$ International Symposium on the Science and Technology of Light Sources, pp.561562 (2004).

(4) Hatta, A. VUV emission spectroscopy of $\mathrm{CO}$ gas discharge, J. Light \& Vis. Env., Vol.29, No.3, pp.79-84 (2005).

(5) Lister, G.G., Lawler, J.E., Lapatovich, W.P., and. Godyak, V.A : The physics of discharge lamp, Reviews of Modern Physics, Vol.76, pp.542-547 (2004).

(6) Nazri, A., Inui, S., Motomura, H., Jinno M., and Aono, M.: Application of ICP in developing mercury-free light sources, IEEJ Trans. FM, Vo.125, No.7, pp.602-607 (2005).

(7) Lindsey, J.L: Applied Illumination Engineering, The Fairmont Press, pp.8-13 (1991).

(8) Mluminating Institute of Japan Editor: Mluminating Engineering, Ohm Publication, p.42 (1983) (in Japanese). 